livraisons

d'Histoire

de l'Architecture

\section{Livraisons de l'histoire de l'architecture}

$37 \mid 2019$

L'architecture mise à l'examen

\title{
La « pédagogie Beaux-Arts » en version américaine : concours et ateliers de la Society of Beaux-Arts Architects
}

The beaux-arts pedagogy in american version: competitions and Workshops of the Society of Beaux-arts architects

Die Beaux-Arts-Pädagogik auf amerikanisch: Wettbewerbe und Ateliers der Society of Beaux-Arts Architects

Isabelle Gournay

\section{OpenEdition}

Journals

Édition électronique

URL : http://journals.openedition.org/lha/1372

DOI : 10.4000/lha.1372

ISSN : 1960-5994

Éditeur

Association Livraisons d'histoire de l'architecture - LHA

Édition imprimée

Date de publication : 15 juin 2019

Pagination : 35-47

ISSN : 1627-4970

Référence électronique

Isabelle Gournay, "La " pédagogie Beaux-Arts » en version américaine : concours et ateliers de la Society of Beaux-Arts Architects », Livraisons de l'histoire de l'architecture [En ligne], 37 | 2019, mis en ligne le 02 janvier 2021, consulté le 25 janvier 2021. URL : http://journals.openedition.org/lha/1372 ; DOl : https://doi.org/10.4000/lha.1372 


\section{LA « PÉDAGOGIE BEAUX-ARTS » EN VERSION AMÉRICAINE : CONCOURS ET ATELIERS DE LA SOCIETY OF BEAUX-ARTS ARCHITECTS}

Les épreuves d'admission au Prix de Rome et, de 1819 à 1968, les concours, sont la clef de voûte de l'enseignement de l'architecture à l'École des Beaux-Arts de Paris. Une transposition particulièrement significative est celle que la Society of Beaux-Arts Architects (SBAA) de New York met sur pied à partir de 1894, à l'intention des dessinateurs d'agence. Calquée sur celle de l'École des Beaux-Arts, l'organisation pédagogique de la SBAA (ill. 1) est finalisée en 1904 avec le Paris Prize, dont le lauréat est admis en première classe de l'École des Beaux-Arts. Ce cursus, qu'administre à partir de 1916 le Beaux-Arts Institute of Design, perdure jusqu’à la fin des années 30. Nous étudions ici la première décennie du $\mathrm{XX}^{\mathrm{e}}$ siècle, quand le système de concours de la SBAA - condensé purement artistique du cursus des Beaux-Arts, autogéré et strictement bénévole - parvient à maturité ${ }^{1}$.

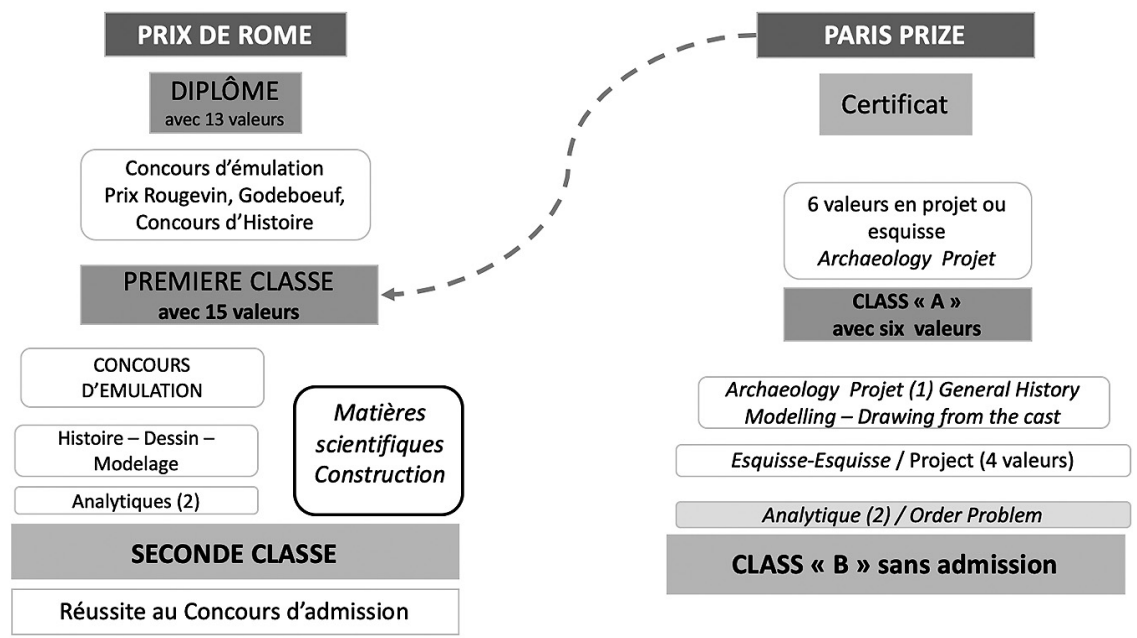

Ill. 1 : Le cursus de l'École des Beaux-Arts (à gauche) et son interprétation par la SBAA. C) Isabelle Gournay.

1. Abréviations : $A A B N$ pour American Architect and Building News.

Cet article s'inscrit dans un projet de recherche sur les Beaux-Arts Architects aux États-Unis. Tout en bénéficiant d'une journée aux archives new-yorkaises du Van Alen Institute, successeur du BAID (https://www.vanalen.org/about/history/), il repose essentiellement sur la consultation de revues spécialisées et de catalogues d'architectural clubs. 


\section{Un contexte favorable}

Le dépôt des statuts de la Society of Beaux-Arts Architects (SBAA) au lendemain de l'Exposition colombienne de Chicago est un jalon majeur de la synergie Beaux-Arts outre-Atlantique. Créée pour "propager" les "principes de bon goût " de l'École des Beaux-Arts parmi la "génération montante des architectes » et le grand public, la SBAA se compose de "membres" statutaires, et d' "associés » ayant fréquenté un atelier parisien pendant au moins un an. Les Américains venant de plus en plus nombreux étudier l'architecture à Paris, la SBAA, dont la cotisation annuelle est modeste, se développe rapidement : en 1907, le dictionnaire d'Edmond Delaire dénombre 130 membres et 20 associés $^{2}$.

Les instigateurs de la SBAA avaient étudié à Paris à la fin des années 1880 ; ils deviennent, et tout particulièrement Whitney Warren et Ernest Flagg, les représentants les plus en vue de l'éclectisme "Modern French ». La conception de leurs édifices aux décors foisonnants et majestueux requiert des centaines de dessins, parfois grandeur nature, et donc des dessinateurs compétents et fiables. Ce sont des jeunes gens ambitieux, se donnant l'image d'artistes pour compenser des salaires inférieurs à ceux des métreurs et surveillants de chantier. En 1905, on estime qu'ils sont 4000 à Manhattan, venus de tout le pays et même de l'étranger ${ }^{3}$.

À Paris, les membres de la SBAA ont pu constater que certains de leurs condisciples français issus de milieux modestes parvenaient au sommet de la hiérarchie des concours de l'École des Beaux-Arts tout en "faisant la place " ${ }^{4}$. Une telle alternance entre atelier et agence est problématique aux États-Unis. Étudier dans une école d'architecture d'obédience Beaux-Arts est trop cher et contraignant; leurs diplômés (une soixantaine par an, alors que 10000 personnes se déclarent architectes aux États-Unis) ont d'ailleurs présenté peu de projets, vue l'importance des cours généraux et obligatoires. Tous aimeraient, à l'instar de leurs employeurs, traverser l'Atlantique pour fréquenter "the École" à laquelle les médias se référent constamment, tant en bien qu'en mal. La SBAA offre donc une alternative gratuite et souple qui, pour quelques élèves, servira de tremplin à un séjour parisien. Instrument de "défense des intérêts communs » de ses membres et ascenseur social, le système de concours de la SBAA reflète, d'une part, la logique capitaliste qui célèbre la Protestant work ethic et les self-made-men et, d'autre part, l'altruisme de la Progressive Era ${ }^{5}$.

2. Edmond Delaire, Les Architectes élèves de l'école des Beaux-Arts, 1793-1907, Paris, Librairie de la Construction Moderne, 1907, p. 447.

3. Mary N. Woods, From Craft to Profession. The Practice of Architecture in Nineteenth-Century America, Berkeley, University of California Press, 1999, p. 146. "Aid for Architects ", Los Angeles Times, 12 décembre 1905, p. 112. Citons l'exemple du Québécois Lucien Kéroack, auteur du Jardin botanique de Montréal, qui remporte le Goelet Prize de la SBAA en 1910.

4. De nombreux fondateurs de la SBAA ont étudié avec Honoré Daumet, dont l'enfance fut pauvre et laborieuse. Voir Henry Roujon, Notice sur la vie et les travaux de M. Honoré Daumet (1826-1911), Paris, Firmin-Didot, 1912, p. 5-6.

5. Paul Nelson, "Courrier des États-Unis. Le Prix de Paris. Propos divers sur l'influence de l'enseignement de l'école aux États-Unis ", L'Architecture 17, 17 septembre 1904, p. 356. 
Notons que l'intense activité des agences peut représenter un handicap pour la SBAA : en 1902, malgré le plus grand nombre d'esquisses préparatoires par rapport à l'année précédente, les "rendus " définitifs ont diminué de 77 à $59^{6}$.

À ses débuts, la SBAA comptait peu de membres parvenus en première classe à l'École des Beaux-Arts. En une décennie, ils sont bien plus nombreux et souvent même diplômés (DPLG). Pour se développer, ce réseau informel a besoin d'un chef d'orchestre possédant qualifications scolaires, disponibilité et entregent. Ce rôle revient à Lloyd Warren (DPLG 1900) ${ }^{7}$. Dès son arrivée à la tête de la commission pédagogique en 1903, le frère cadet de Whitney Warren organise une campagne d'information dans les revues spécialisées. Les résultats sont probants: la SBAA juge 184 soumissions en 1903, 436 en 1904 ; elle compte près de mille participants en 1912, chiffre remarquable si on le compare aux 965 élèves architectes que Delaire répertorie à l'École des Beaux-Arts. Lloyd Warren élabore un règlement interne et institue un certificat de fin d'études; dans son hôtel particulier de la Cinquième Avenue, il organise des conférences mondaines au profit de la SBAA. Avant qu'il ne décède en 1922, il sera la cheville ouvrière du Beaux-Arts Institute of Design (BAID) et de l'American E.F. Training Center of Art de Meudon-Bellevue, destiné aux soldats américains en attente de rapatriement et prélude aux Écoles d'art américaines de Fontainebleau.

\section{La participation des ateliers, clubs et programmes universitaires}

À l'instar de l'École des Beaux-Arts, la pédagogie de concours de la SBAA est destinée à être menée dans des ateliers indépendants, où des élèves débutants et confirmés travaillent de concert et s'entraident. Un tel mimétisme résulte, d'une part, de l'admiration que les Beaux-Arts Architects portent aux chefs d'ateliers parisiens, pour la manière dont ces derniers concilient enseignement et pratique, et d'autre part, d'une mystique folklorique, quasi-ethnographique, de l'atelier parisien qu'entretiennent certains membres de la $\mathrm{SBAA}^{8}$. Les termes de "patron" ou de "massier" présidant aux contingences matérielles de l'atelier ne sont pas traduits en anglais. Lofts au confort rudimentaire, les ateliers de Midtown Manhattan sont proches des agences. Dépassant rarement la trentaine, leurs effectifs sont suffisamment modestes pour que les débutants puissent recevoir des corrections du patron, et non d'assistants comme dans les ateliers parisiens les plus prestigieux. Les résultats de concours publiés dans la presse spécialisée indiquent que, comme à Paris,

6. "Society of Beaux-Arts Architects - Report of Committee on Education ", $A A B N$ 79, 21 février 1903, p. 63.

7. Lloyd Warren préside la commission pédagogique de 1903 à 1906 et en 1908-09; il en fait partie en 1909-10 et préside le conseil d'administration en 1907-08.

8. Par exemple, le fils du grand romancier William Dean Howells, John Mead Howells (DPLG 1897) publie "From Nouveau to Ancien at the École des Beaux-Arts ", Architectural record, no spécial, janvier 1901, p. 35-55 et "A French Government School From The Inside », Century Illustrated Magazine, octobre 1901, p. 864-68. 
quelques ateliers monopolisent les récompenses. Vers 1903, les patrons les plus couronnés de succès sont Henry Hornbostel, dont les talents de perspectiviste sont reconnus de part et d'autre de l'Atlantique ${ }^{9}$, Joseph H. Freedlander, diplômé en 1895 et dont l'atelier ouvre en 1896 et Donn Barber (DPLG 1898) ${ }^{10}$.

La création d'ateliers au-delà de New York reflète le dynamisme de la " diaspora " des Beaux-Arts outre-Atlantique. Dès 1902, Theodore Pietsch (DPLG 1897) en organise un à Washington : la demande y est forte parmi les employés du Supervising Architect of the Treasury, où lui-même travaille à la conception d'édifices fédéraux. La SBAA est représentée à Chicago par Edward H. Bennett (DPLG 1901) ; à Cleveland par J. Milton Dyer (DPLG 1900) et à Cincinnati par Albert Fechheimer (DPLG 1904). Ce mouvement gagne le Canada, où William S. Maxwell et John Lyle ouvrent des ateliers, à Montréal et Toronto respectivement.

La formule de l'atelier se pérennise au sein des architectural clubs, dont les locaux sont plus confortables et où des causeries complètent le cursus de la SBAA ${ }^{11}$. Le San Francisco Architectural Club parraine trois ateliers, dirigés par John Baur (DPLG 1901), Loring P. Rixford et Arthur Brown (DPLG 1901) en tandem avec son collaborateur Jean-Louis Bourgeois. Afin de réduire les frais et les délais encourus en faisant juger les projets de la côte ouest à New York, la SBAA ouvre une antenne à San Francisco. Ses sujets de concours sont également repris par les programmes universitaires ; en 1912, les trois quarts d'entre eux les suivent de manière plus ou moins régulière. Un jugement à l'échelle nationale bénéficie particulièrement aux programmes nouvellement créés, tel que Georgia Tech à Atlanta. À Saint-Louis, Washington University accueille alternativement des étudiants à temps plein et des dessinateurs, tous supervisés par Louis Spiering (DPLG 1902).

\section{Un condensé purement artistique du cursus Beaux-Arts}

Persuadés de son excellence, les membres de la SBAA reproduisent le système de concours qu'ils ont suivi à Paris, tout en éliminant les éléments qui semblent superflus, voire préjudiciables, pour des dessinateurs : en particulier, on n'impose ni concours d'admission, ni limite d'âge. Le calendrier tient compte de l'emploi à plein temps des élèves : les concours commencent le samedi et sont rendus un lundi. Comme à Paris, le maintien de l'inscription est peu contraignant car il suffit de présenter trois dessins dans l'année. Tout en conservant la hiérarchie entre projets simples et plus complexes, les première et seconde classes sont rebaptisées Class A (Advanced) et B (Beginners). La SBAA élimine entièrement l'enseignement scienti-

9. Francis S. Swales, « Master draftsmen XVII, Henry Hornbostel », Pencil Points, vol. 7, février 1926, p. 73-92.

10. Maurice Prévot, Second Grand Prix de Rome en 1902, dirige également un atelier : voir Isabelle Gournay, « De Paul Cret à Jean Labatut, la contribution des French critics aux États-Unis », Rayonnement des écoles et relations internationales HEnsA20, 2018, https ://chmcc.hypotheses.org/4304.

11. À Minneapolis, l'architectural club accueille l'atelier qu'Edwin H. Hewitt (diplômable en 1904) avait préalablement organisé dans sa propre agence. 
fique; sans doute la manière dont ces matières sont étudiées à Paris paraît trop sophistiquée et théorique pour des dessinateurs, qui peuvent en apprendre les principes essentiels dans des établissements philanthropiques comme la Cooper Union à New York.

Si elle requiert moitié moins de points, la séquence "d'architecture » est similaire. Deux order problems, équivalents aux concours sur éléments analytiques, sont obligatoires avant de présenter des plan problems de plusieurs semaines. Mise à part pour le sketch problem (également nommé esquisse-esquisse) une esquisse préliminaire est obligatoire. Dans les locaux de la rue Bonaparte, l'esquisse en loges dure 12 heures, durée que la SBAA réduit à 9 heures. La SBAA adopte le principe irrévocable du Hors de concours pour toute esquisse qui n'a pas été effectuée en loge et projet rendu sans soin.

Le cursus de la SBAA requiert deux valeurs d'archaeology sanctionnées par des concours ouverts aux deux classes. Le certificat de fin d'étude requiert, comme en seconde classe à Paris, une valeur de modelage et une de dessin, que les New-Yorkais sont enjoints de prendre (à frais d'études réduits) à l'Art Student League. On doit également réussir un examen écrit et oral d'histoire générale, inspiré de celui pour l'admission à l'École des Beaux-Arts. Les modalités de rendu en deux dimensions au lavis ou à l'aquarelle et de jugement à huis clos sont aussi transplantées depuis Paris. Toutefois l'exposition publique de tous les projets a lieu la veille du jury à New York alors qu'elle lui est postérieure et de plus longue durée à l'École des Beaux-Arts. À New York comme à Paris, on juge plusieurs concours à une cadence rapide, en distribuant mentions et médailles valant un certain nombre de points, puis en établissant un classement nominatif au sein de chaque catégorie ${ }^{12}$. Certes il n'existe pas en Amérique d'imposants folios de "concours de l'année scolaire " comme ceux qu'éditent Farge, Guérinet ou Vincent, et les comptes rendus journalistiques n’ont ni la régularité ni la pertinence de ceux de la Construction Moderne. Toutefois les occasions d'exposer ses dessins scolaires existent autant au sein des expositions annuelles des architectural clubs que dans les Salons parisiens.

À l'École des Beaux-Arts, les programmes des concours d'émulation sont rédigés par le professeur de Théorie, une tâche dont Julien Guadet s'acquitte, de 1894 à 1908 , avec une grande diligence ${ }^{13}$. Les membres de la SBAA se partagent cette même responsabilité. Par conviction ou nostalgie, ils reprennent parfois un sujet

12. À la SBAA, les esquisses-esquisses des deux classes, ainsi que le plan problem en Class B peuvent recevoir une seconde mention ( $1 / 2$ point) or une première mention ( 1 point) ; le plan problem en class A une première mention ( 1 point); une seconde médaille ( $11 \frac{1}{2}$ point) et une première médaille ( 2 points). Les médailles en bronze sont gravées par le sculpteur et médailliste d'origine française Jules Édouard Roiné. Nous n’avons pas encore trouvé de trace de la " courte notice " que chaque concurrent était censé recevoir du jury de la SBAA. Les commentaires du jury accompagnent parfois la publication des projets lauréats et du classement dans les revues d'architecture.

13. Le programme du concours de 1895 pour un grand magasin, illustré dans Joan Oakman (sous la dir. de), Architecture School. Three Centuries of Educating Architects in North America, Cambridge, MIT Press, 2012, p. 277, imite les dispositions graphiques des programmes de l'École des BeauxArts, que de nombreux anciens élèves américains ramènent de Paris. 
sur lequel ils ont concouru à Paris ${ }^{14}$. Rares sont ceux qui signalent clairement les attentes du jury, comme y parvient Lloyd Warren, qui stipule pour un asile de nuit une "composition de caractère extrêmement simple" où l'effet architectural serait obtenu par «la couleur, les matériaux et le profil des masses " ${ }^{15}$. Le concours sur éléments analytiques est ajouté au cursus de l'École des Beaux-Arts à la fin des années 1870. Guadet l'envisage comme un exercice didactique, une transition entre esquisse d'admission et projets rendus : l'élève ne doit pas " copier des détails dans des livres " mais intérioriser la " grammaire première " des "éléments d'architecture " héritée de l'Antiquité et de la Renaissance ${ }^{16}$. Plus narratif, l'order problem de la SBAA s'apparente davantage aux compositions de "fragments antiques" produite par les Grands Prix de Rome ${ }^{17}$. Une étude de frontispice (ill. 2) requiert que les "détails classiques soient disposés d'une manière attrayante": en plus d'une colonne d'au moins deux pouces de diamètre, il faut inclure « un chapiteau corinthien de Pompéi, un autel romain, une stèle grecque, des masques comique et tragique, un grand vase d'ornement, un morceau de mosaïque, une peinture murale pompéienne, un tripode, des guirlandes de laurier, une acrotère, un fragment de frise ou bas-relief comprenant chevaux ou personnages, du mobilier pompéien ${ }^{18}$. De tels exercices, où le langage classique revêt une dimension plus éclectique que rationaliste, servent certainement d'échappatoire à la routine de l'agence.

Le concours d'archaeology est conçu pour "pouvoir différencier les époques" en établissant un "une composition originale selon un style reconnu ", en général d'origine française $^{19}$. Il reprend la formule du concours d'histoire de l'architecture de première classe à l'École des Beaux-Arts. L'épreuve de 1901, "Une chaire dans une Église du XIII ${ }^{\mathrm{e}}$ siècle» (où Spiering s'était classé premier) devient "A Pulpit in the style of the XIIIth Century " (ill. 3). Mais tandis que le programme rédigé par Lucien Magne est précis et érudit, sa version américaine est peu précise sur la manière dont "se conformer au style de l'époque".

Les sujets de concours reflètent parfois le mouvement d'embellissement urbain City Beautiful, mais sans indication de site précis. Une esquisse-esquisse en Class A prescrit l'insertion d'un Monument à Robert Fulton dans " une niche d'un des murs de soutènement de la route longeant l'Hudson ${ }^{20}$. Un gymnase avec loggia, un monument au généreux donateur, ainsi que des portillons décoratifs sont prescrits pour transformer un îlot new-yorkais de 600 par 200 pieds en un « oasis dans

14. En 1905, J. Layng Mills remporte une première mention en seconde classe (projet rendu) pour "La Paroi du fond d'une cage de grand escalier"; en 1910, il propose pour la Class B "Grand Stairway In a Court House."

15. Architecture 11, 15 janvier 1905, p. 17 (illustrations, p. 16).

16. Julien Guadet, Éléments et théorie de l'architecture, Paris, Aulanier, 1901, t. 1, p. 92.

17. Hector d'Espouy, Fragments d'architecture Antique d'après Les Relevés \& Restaurations Des Anciens Pensionnaires de l'Académie de France à Rome, Paris, C. Schmid, 1890.

18. Architecture 11, 15 mai 1905 , p. 84.

19. $A A B N 87,21$ janvier 1905, p. vi.

20. Season of 1909-1910, Year-book of premeated designs illustrating the educational work conducted by the Society of Beaux-Arts Architects..., New York, From the publishers of : American Architect, 1910, p. 15. 


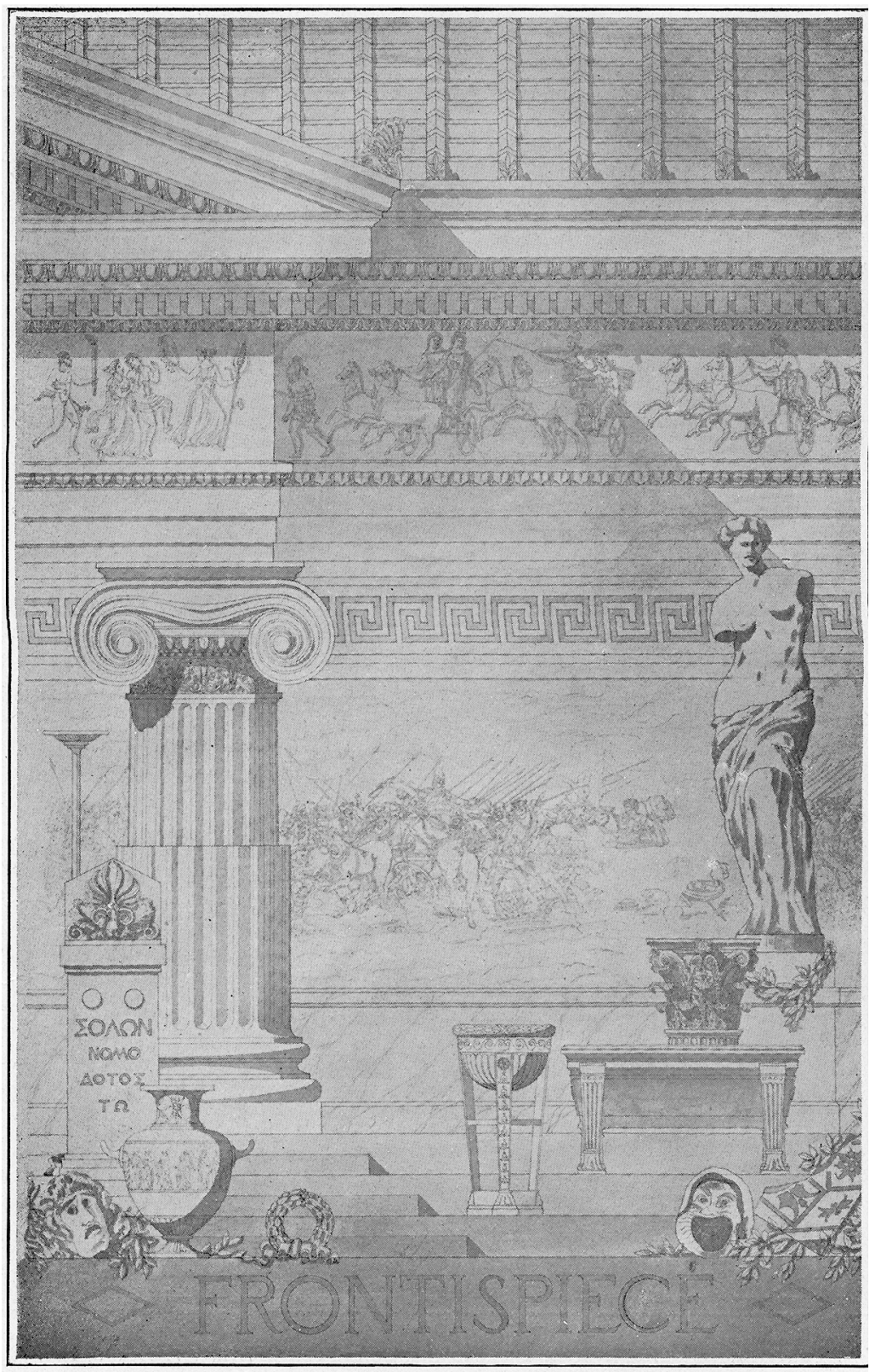

A FRONTISPIECE.

Beaux Arts Society Competition.

GeO, H. MATTHEWS, WAShINGTON ATELIER.

Ill. 2: Geo H. Matthews, Washington Atelier, A Frontispiece, Class B Order Problem (Analytique) (Catalogue of the fifth exhibition of the Washington Architectural Club, 1905). (C) D.R. 


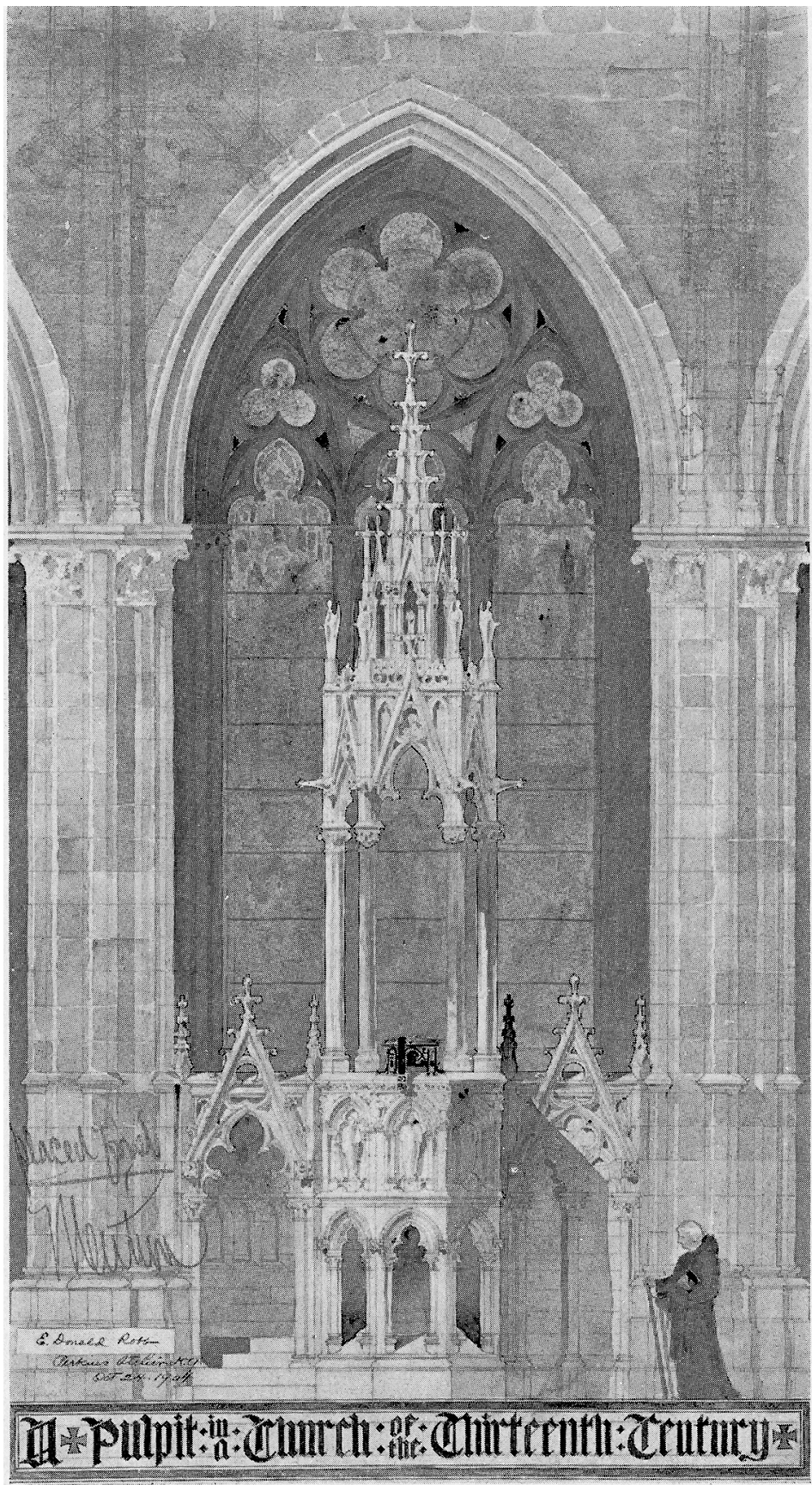

Ill. 3 : E. Donald Robb, Atelier Blair \& Van Pelt (New York), A Pulpit in the style of the XIIIth Century, Archaeology (T Square Club, Catalogue of the eleventh annual exhibition, 1905). () D.R. 


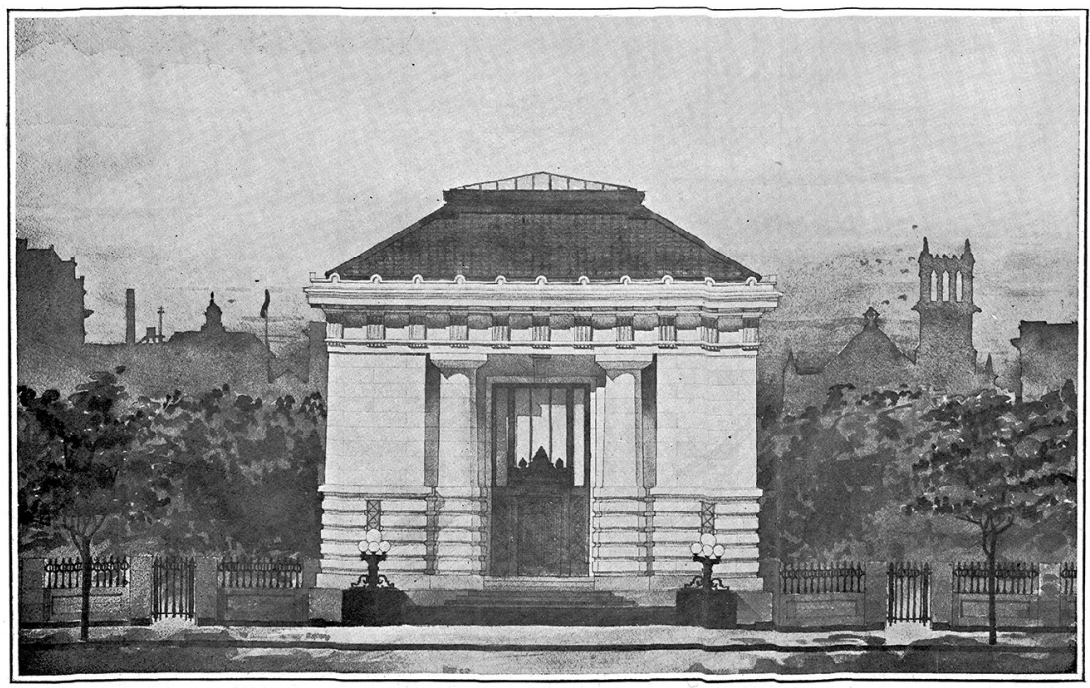

Ill. 4 : Norman T. Vorse, Atelier Pietsch (Washington), Une petite Banque - Plan Problem, Classe B (Catalogue of the fifth exhibition of the Washington Architectural Club, 1905). (C) D.R.

le désert de maisons et immeubles de bureaux en briques " ${ }^{21}$. On note la rareté des sujets de décors intérieurs (très présents à l'École des Beaux-Arts), l'absence de gratte-ciel et la rareté des édifices commerciaux, à moins qu'il ne s'agisse d'une banque (ill. 4) qui revêt dans toute ville américaine un caractère semi-public. L'architecture des transports (gares et ferrys) est très en vogue, comme à l'ENSBA. Quelques sujets sortent de l'ordinaire, tel cette esquisse-esquisse en Class B pour un "pavillon d'électrocution pour les détenus d'une prison d'état » ${ }^{22}$. De nombreux sujets de concours se rapportent au mode de vie des élites. Pour une gare de banlieue (ill. 5) " conçue en harmonie avec ses abords pittoresques " le programme fait allusion à l'enclave ultra-chic de Tuxedo Park aux environs de New York, où plusieurs membres de la SBAA ont exercé leur talent ${ }^{23}$. Les commentaires des jurys traduisent la difficulté qu'ont les élèves dessinateurs de décrypter un monde qu'ils ne connaissent qu'à travers des lectures ou les commandes de leur agence; par exemple, on critique la manière dont le casino d'un country club «dans une station estivale à la mode " ressemble à Luna Park ou à Monte Carlo ${ }^{24}$. Aux mains des élèves les plus capables, les esquisses-esquisses de Class A (ill. 6) ressemblent aux "petits ensembles lestement trouvés, habilement rendus" des concours du même types donnés à l'École des Beaux-Arts ${ }^{25}$. Leur rendu perspectif et atmosphérique échappe aux conventions Beaux-Arts.

21. "Esquisse-esquisse Class B ", Architecture 9, 15 avril 1904, p. 61-62.

22. Achitecture 12, 15 juillet 1905, p. 115 .

23. Architecture 11, Septembre 1904, p. 146.

24. $A A B N$ 97, 11 mai 1910, p. 3.

25. Julien Guadet, Éléments et Théorie, op. cit., p. 93. 


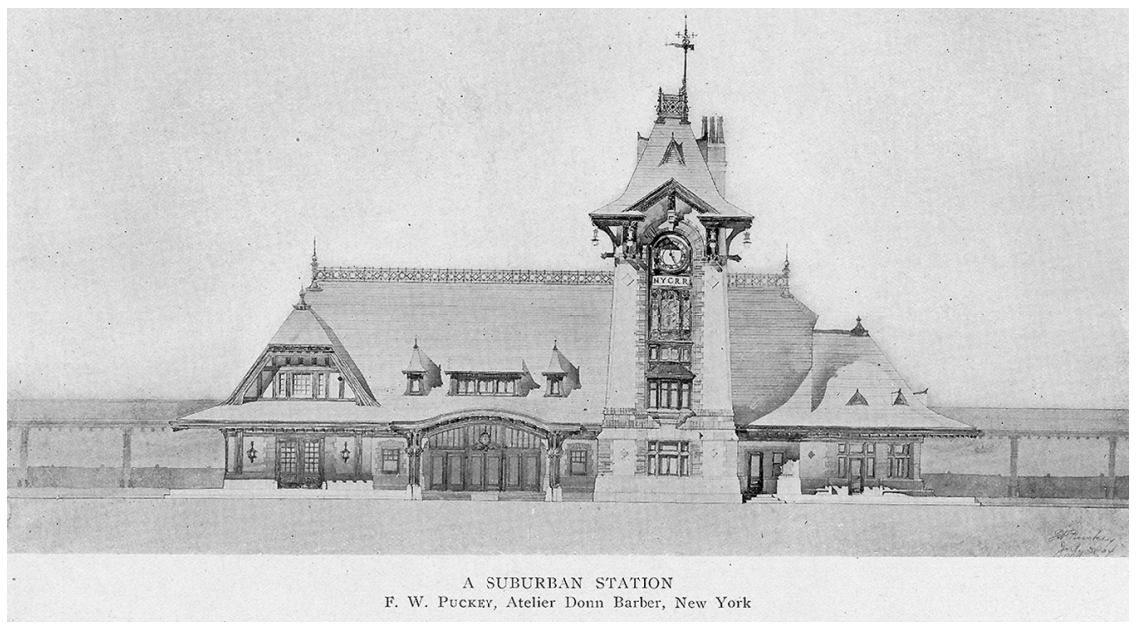

Ill. 5 : Francis Willard Puckey, Atelier Donn Barber, New York, Gare suburbaine (T Square Club, Catalogue of the eleventh annual exhibition, 1905). (C) D.R.

L'esquisse-esquisse (ill. 6) que nous reproduisons est l'œuvre de John Wynkoop, second lauréat du Paris Prize, dont la création marque une étape déterminante pour la SBAA. Si on la compare à l'École des Beaux-Arts ou aux écoles d'architecture américaines, elle offre peu de récompenses monétaires. Le Warren Prize s'inspire du concours Labarre ; il est offert par Whitney et Lloyd Warren, dont le neveu Robert Goelet finance un prix pour la planification d'un îlot urbain ${ }^{26}$. En Class A, "le concurrent sérieux" ne peut que "cumuler les médailles, sans autre sanction que l'amour de l'art " ${ }^{27}$. Pour raviver "l'ardeur de la lutte " la SBAA envisage une bourse de voyage aussi avantageuse que les bourses de voyage Rotch, Stewardson et McKim, ou un séjour à l'American Academy de Rome. Le bénéficiaire aurait séjourné deux ans en Europe, dont au moins huit mois dans un atelier parisien pour tenter l'admission à l'École des Beaux-Arts. On imite la sélection en trois étapes du concours de Rome : esquisse de 12 heures à New York et dans huit autres villes, ouverte à toute personne de moins de 28 ans; esquisse de 24 heures pour les cinq finalistes du premier tour et les 15 élèves les plus compétitifs de la SBAA; les cinq logists s'attaquent enfin à un projet de grande ampleur, soumis à un jury plus étoffé que celui des concours ordinaires. Au printemps 1904, George A. Licht remporte cette course d'obstacles. Depuis 1896, il travaille dans l'agence de Freedlander et étudie dans son atelier ; il s'est déjà présenté aux épreuves d'admission de l'École des Beaux-Arts, où son esquisse avait reçu la meilleure note ${ }^{28}$. Grâce à

26. Les sommes varient de 15 à 50 dollars (cette dernière somme correspond à $1300 \$$ actuels).

27. Paul Nelson, "Courrier des États-Unis", L'Architecture, op. cit., p. 356. Vers 1910, le Bacon Prize offre de 40 à 100 dollars pour les élèves ayant cumulé un maximum de points en Class A.

28. Intitulée «A Monument to Commemorate Illustrious Men (Design placed First, Entrance Examination Beaux Arts School, Paris " cette esquisse est exposée au T-Square et au Washington Architectural Clubs. 


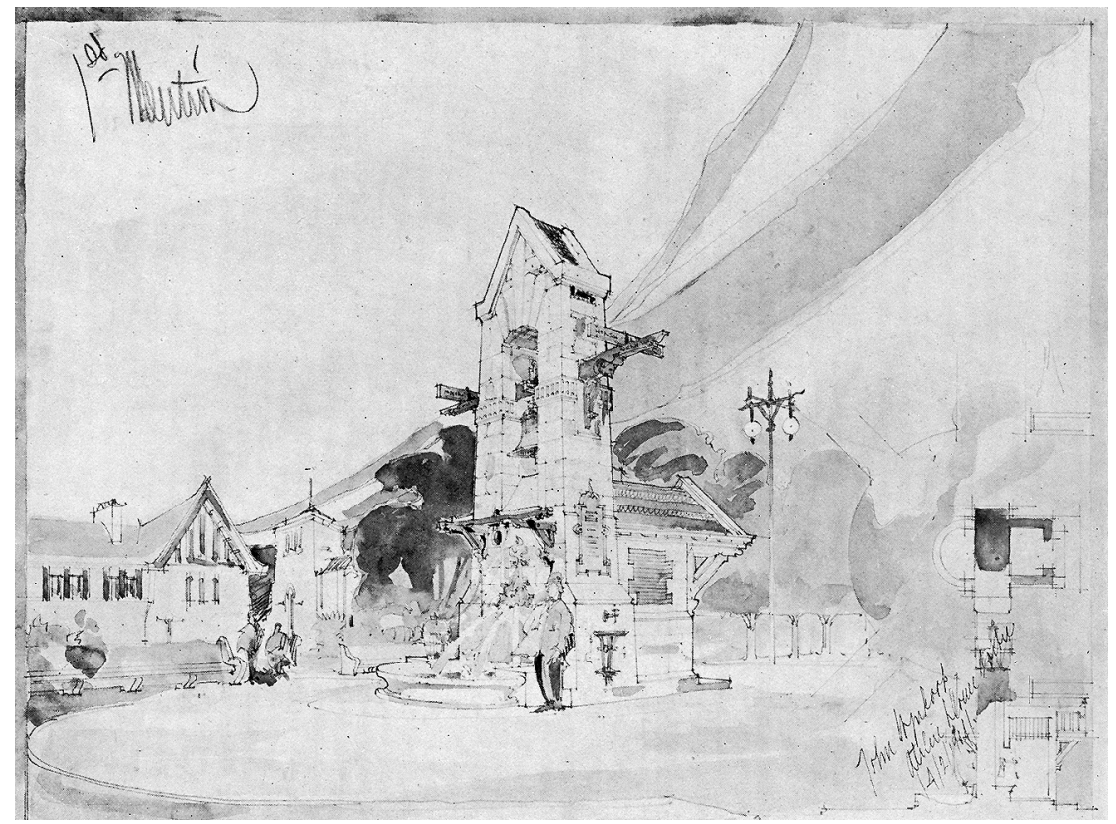

Ill. 6 : John Wynkoop, Atelier Donn Barber, A Market Cross, Esquisse-Esquisse Class A (T Square Club, Catalogue of the eleventh annual exhibition, 1905). (C) D.R.

l'intervention directe de Lloyd Warren (accompagné de Louis Bernier) auprès d'un comité composé de Guadet, Vaudremer, Pascal, Nénot et Moyeux, le lauréat du Paris Prize reçoit l'autorisation tout à fait exceptionnelle de s'inscrire directement en première classe, tout en ne pouvant pas présenter de diplôme ou bénéficier de récompenses monétaires ${ }^{29}$. Licht reçoit en 1906 la Grande Médaille d'émulation, se haussant au niveau des "grands élèves" français qui volent de médaille en médaille ${ }^{30}$. Un tel succès permet à la SBAA de solliciter un financement de la part de millionnaires très connus : c'est ainsi qu'Andrew Carnegie sponsorise Wynkoop et Pierpont Morgan le troisième lauréat, Frederick Hirons (ill. 7) ${ }^{31}$.

29. C'est en mars 1905 que le Conseil supérieur entérine l'arrêté du ministre de l'Instruction publique et des Beaux-Arts de décembre 1904. Le règlement de l'École des Beaux-Arts n'indique pas nommément la SBAA mais autorise " un Prix de Paris » qui doit être organisé par une société « composée exclusivement d'anciens élèves de l'École nationale des Beaux-Arts ou des Écoles régionales d'architecture de France et comprendre cent membres au minimum ». Le lauréat doit "faire preuve de connaissances équivalentes à celles acquises en seconde classe ».

30. La feuille de valeurs de Licht est consultable sur la base de données " Dictionnaire des élèves architectes de l'École des Beaux-Arts (1800-1968) ", en ligne sur le site de l'Institut national d'histoire de l'art (INHA).

31. Sur Hirons admis par "voie normale" à l'École des Beaux-Arts en avril 1905 mais reparti dès octobre de la même année par manque de moyens financiers, voir Isabelle Gournay et Marie-Laure Crosnier Leconte, "American Architecture Students in Belle Époque Paris: Scholastic Strategies and Achievements at the Ecole des Beaux-Arts ", The Journal of the Gilded Age and Progressive Era 12, avril 2013, p. 168-170. 


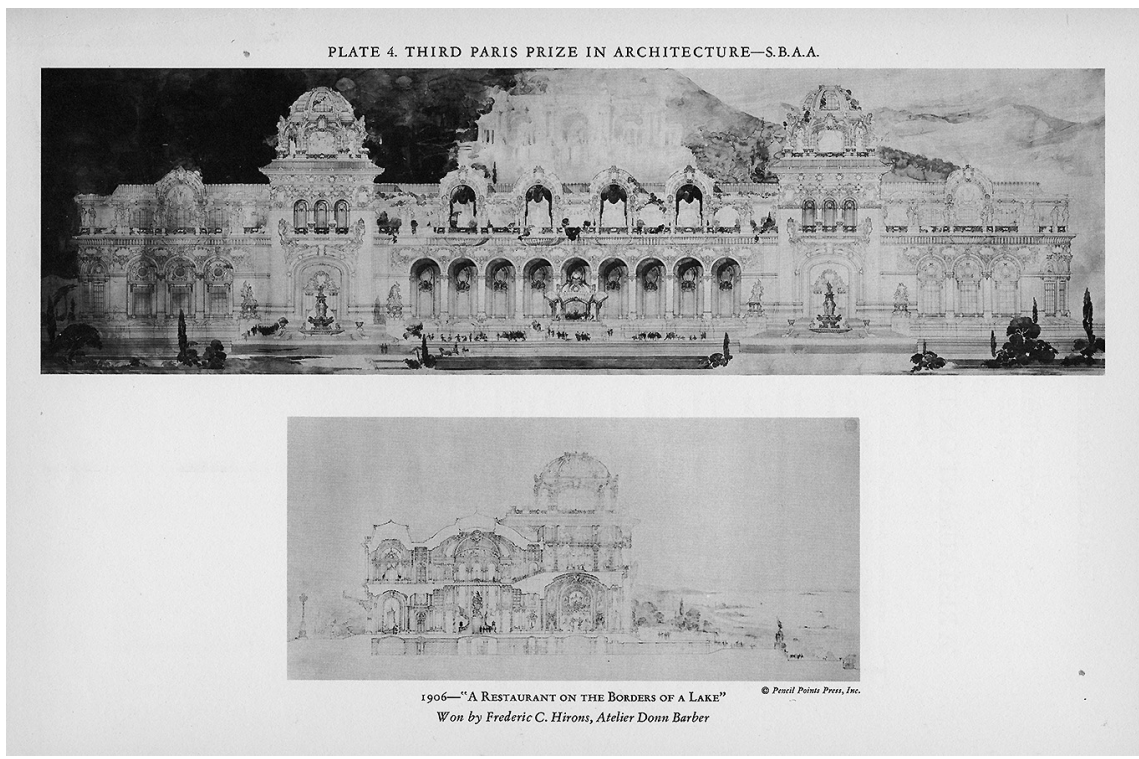

Ill. 7 : Frederic Hirons, Un restaurant au bord d'un lac, Projet lauréat du Paris Prize, 1906 (Winning designs, 1904-1927: Paris prize in architecture, Lloyd Warren Memorial, 1928). () D.R.

Le système de concours de la SBAA a bien entendu les défauts de ses qualités. L'autonomie institutionnelle, la souplesse de fonctionnement et le bénévolat de praticiens de premier plan encourent un fort risque de volatilité et les ateliers affiliés à la SBAA n’ont pas la longévité de ceux de Paris. Ce système né à New York, se nourrissant du dynamisme économique et artistique de cette métropole mondiale, devient précaire dans une ville moyenne, où ne s'implique parfois qu'un seul architecte. À l'époque où la France crée des écoles d'architecture en province, l'ambition centralisatrice de la SBAA à l'échelle des États-Unis et du Canada peut paraître chimérique : ce système n'est efficace que parce que les effectifs d'élèves et d'enseignants sont encore modestes.

Vers 1910, la SBAA a rempli les objectifs pédagogiques qu'elle s'était fixés à son origine; avec le "court-circuitage" du cursus de l'École des Beaux-Arts (voir ill. 1) obtenu pour les lauréats de son Paris Prize, elle les a même surpassés. La formule de l'atelier affilié à la SBAA a été un catalyseur artistique pour ceux dont le parcours personnel et professionnel n'est pas linéaire. Il a également contribué à une plus grande diversité ethnique parmi les architectes américains : alors que les admis en seconde classe de l'École des Beaux-Arts sont pour la plupart anglo-saxons et protestants, les palmarès de la SBAA comprennent beaucoup de noms à consonance italienne ou slave, qui sont le plus souvent émigrants de fraiche date. Ces palmarès indiquent en de rares occasions un prénom féminin, et certaines initiales pourraient être celles de concurrentes ; toutefois ces jeunes femmes ont été admises dans un programme universitaire (dont MIT, Cornell et Berkeley). Les ateliers 
cultivent un folklore masculin, voire machiste et le cursus de la SBAA encourage un comportement qu'on peut qualifier de viril : maintenir la pression des esquisses en loge ou des charrettes s'apparente à l'exploit sportif que la civilisation américaine célèbre parmi ses jeunes hommes.

Parmi ses détracteurs les plus acharnés se trouvent quelques professeurs d'architecture exerçant dans un cadre universitaire. À Columbia, William Robert Ware dénonce les carences intellectuelles d'un système qui, selon lui, néglige les humanités et fétichise l'expression graphique. Écossais enseignant à McGill, Percy Nobbs voit dans cette version new-yorkaise des Beaux-Arts un dangereux vecteur d'américanisation; les concours d'archaeology abâtardissent selon lui les sources d'inspiration " naturelles et légitimes» que l'architecture du Moyen-Âge et de la Renaissance en France et en Grande-Bretagne représente pour le Canada ${ }^{32}$. Les objections sont également d'ordre pratique : à Berkeley, Warren Perry indique que les calendriers de son école et celui de la SBAA sont incompatibles; il trouve les énoncés des programmes " ambigus» et mentionne des prescriptions d'échelle erronées ${ }^{33}$. Toutefois beaucoup de programmes universitaires bénéficient de ce système de concours, où leur petit contingent d'étudiants peut se mesurer, à l'échelon national, à des dessinateurs plus âgés et expérimentés.

A posteriori, nous comprenons que le système académique français exerce une influence sur les architectes américains bien au-delà de ceux qui, autant par privilège social que par talent, ont été admis à l'École des Beaux-Arts. Si le cursus et les méthodes de la SBAA ont pu inculquer chez certains une habileté superficielle, acquise en intégrant des codes et en reproduisant des modèles parfois surannés, elles ont manifestement produit des talents originaux qui se sont illustrés dans l'Art Déco et des architectes de stature régionale ou nationale dont le travail n'a été ni banal, ni conventionnel.

\section{Isabelle GOURNAY \\ University of Maryland (Emerita)} et AHTTEP, École nationale supérieure d'architecture de Paris-La Villette

32. Kelly Crossman, Architecture in transition : From art to practice, 1885-1906, Kingston Ont. : McGillQueens University Press, 1987, p. 129.

33. Warren C. Perry, "The Teaching of Architecture on the Pacific Coast ", Architect and Engineers of California 34, octobre 1913, p. 67. 
